\title{
Littlewood-Paley Functions and Triebel-Lizorkin Spaces, Besov Spaces
}

\author{
Dashan Fan ${ }^{1}$ and Fayou Zhao ${ }^{2 *}$ \\ ${ }^{1}$ Department of Mathematical Sciences, University of Wisconsin-Milwaukee, \\ Milwaukee, WI 53201, USA \\ 2 Department of Mathematics, Shanghai University, Shanghai 200444, China
}

Received 7 May 2020; Accepted (in revised version) 21 July 2021

Dedicated to Prof. Shanzhen Lu with admiration on the occasion of his 80th birthday

\begin{abstract}
We establish Littlewood-Paley charaterizations of Triebel-Lizorkin spaces and Besov spaces in Euclidean spaces using several square functions defined via the spherical average, the ball average, the Bochner-Riesz means and some other wellknown operators. We provide a simple proof so that we are able to extend and improve many results published in recent papers.
\end{abstract}

Key Words: Littlewood-Paley square functions, Triebel-Lizorkin spaces, Besov spaces, spherical average, ball average, Bochner-Riesz means.

AMS Subject Classifications: 46E35, 42B25, 42B35

\section{Introduction}

As is well known, Littlewood-Paley functions and their various applications are important parts of harmonic analysis, dating back as far as the early 1930's; see [10, 19,20,22] for more details about the historical development. Recently Alabern et al. in [1] obtained a new characterization of Sobolev spaces with arbitrary smoothness order on Euclidean spaces, which can be seen as characterizations of Sobolev spaces via LittlewoodPaley $g$-functions involving ball averages. These characterizations depend only on the metric of $\mathbb{R}^{n}$ and hence provide several possible approaches to introduce high order Sobolev spaces on general metric measure spaces. Motivated by the work in [1], some characterizations of high order Besov and Triebel-Lizorkin spaces on $\mathbb{R}^{n}$ in terms of Littlewood-Paley functions and pointwise inequalities involving ball averages were further established, which also serve as new approaches to introduce these spaces with high order smoothness on metric measure spaces. Yang et al. in [26] established the corresponding characterizations for Besov and Triebel-Lizorkin spaces. Inspired by [1,26],

*Corresponding author. Email addresses: fan@uwm.edu (D. Fan), fyzhao@shu . edu.cn (F. Zhao) 
Dai et al. further characterized Sobolev spaces with any positive even integer order via some pointwise inequalities involving ball averages in [6], as well as Besov and TriebelLizorkin spaces with any positive smoothness order via some Littlewood-Paley $g$ functions involving ball averages in [7]. Based on [7], Chang et al. in [3] considered the related characterizations of Triebel-Lizorkin spaces via the corresponding Lusin area function and the Littlewood-Paley $g_{\lambda}^{*}$-function. Some further characterizations of Sobolev, Besov and Triebel-Lizorkin spaces via ball averages were then presented in a series of works $[4,8,13,15,25,28-30]$.

One the other hand, Chen et al. in [5] gave a simple method to characterize inhomogeneous Sobolev spaces $W^{\alpha, p}\left(\mathbb{R}^{n}\right)$ by using several different square functions defined via the spherical average, the ball average and the Bochner-Riesz means. Based on the aforementioned works, the main purpose of this article is to characterize Triebel-Lizorkin and Besov spaces via some generalized Littlewood-Paley functions which are much more general than those Littlewood-Paley functions of ball averages. We extend their results, using an alternate, less complicated method of proof.

To this end, we firstly give some necessary notations. Let $n \geq 2$ and $\mathbb{R}^{n}$ be $n$ dimensional Euclidean space. Fix an $L^{1}\left(\mathbb{R}^{n}\right)$ function $\Phi$. Denote, for $(x, t) \in \mathbb{R}^{n} \times \mathbb{R}$,

$$
\Phi_{2^{t}}(x)=2^{-t n} \Phi\left(x / 2^{t}\right) .
$$

The Fourier transform of $\Phi_{2^{t}}$ is given by $\widehat{\Phi_{2^{t}}}(\xi)=\widehat{\Phi}\left(2^{t} \xi\right), \xi \in \mathbb{R}^{n}$. For any $1<q<\infty$, associated with $\Phi$, the Littlewood-Paley function $S_{\Phi, q}(f)$ is defined by

$$
S_{\Phi, q}(f)(x)=\left(\int_{\mathbb{R}}\left|\Phi_{2^{t}} * f(x)\right|^{q} d t\right)^{1 / q}
$$

and the discrete version is given by

$$
D_{\Phi, q}(f)(x)=\left(\sum_{k \in \mathbb{Z}}\left|\left(\Phi_{2^{k}} * f\right)(x)\right|^{q}\right)^{1 / q} .
$$

Sometimes we write $S_{\Phi, q}(f)(x)$ in an equivalent form

$$
S_{\Phi, q}(f)(x)=\left(\int_{0}^{\infty}\left|\Phi_{t} * f(x)\right|^{q} \frac{d t}{t}\right)^{1 / q},
$$

and skip the ratio $(\ln 2)^{-1 / q}$ between two forms. Also, for simplicity, we initially define $S_{\Phi, q}(f)$ on all functions $f$ in the Schwartz space $\mathcal{S}\left(\mathbb{R}^{n}\right)$. Let

$$
\Delta=\sum_{j=1}^{n} \frac{\partial^{2}}{\partial x_{j}^{2}}
$$


be the Laplace operator. Taking the Fourier transform, we have

$$
-\widehat{\Delta(f)}(\xi)=4 \pi^{2} \sum_{j=1}^{n} \xi_{j}^{2} \widehat{f}(\xi)=4 \pi^{2}|\xi|^{2} \widehat{f}(\xi)
$$

For a complex exponent $z$, the fractional Laplacian $(-\Delta)^{z / 2}$ is defined, via the Fourier transform, by

$$
(-\Delta)^{z / 2}(f)(x)=\left((2 \pi|\xi|)^{z} \widehat{f}(\xi)\right)^{\vee}(x) .
$$

The inhomogeneous Sobolev space $W^{\alpha, p}\left(\mathbb{R}^{n}\right)$ of smoothness order $\alpha \in \mathbb{R}$, for a fixed $p \in[1, \infty)$, is the set of all functions $f$ satisfying

$$
\|f\|_{W^{\alpha, p}\left(\mathbb{R}^{n}\right)}=\|f\|_{L^{p}\left(\mathbb{R}^{n}\right)}+\left\|(-\Delta)^{\alpha / 2} f\right\|_{L^{p}\left(\mathbb{R}^{n}\right)}<\infty .
$$

The Sobolev space plays a significant role not only in the theory of partial differential equations, but also in many other fields, such as functional analysis and harmonic analysis. Let $B(x, t)$ be the ball centered at $x$ with radius $t>0$. For any locally integrable function $f$, define the average of $f$ over the ball $B(x, t)$ by

$$
B_{t}(f)(x)=\frac{1}{|B(x, t)|} \int_{B(x, t)} f(y) d y,
$$

where $|B(x, t)|$ denotes the volume of $B(x, t)$. In [1], Alabern, Mateu and Verdera studied two square functions

$$
\begin{aligned}
& T_{\alpha, 2}(f)(x)=\left(\int_{0}^{\infty}\left|\frac{B_{t}(f)(x)-f(x)}{t^{\alpha}}\right|^{2} \frac{d t}{t}\right)^{1 / 2}, \\
& \mathcal{G}_{\alpha, 2}(f, g)(x)=\left(\int_{0}^{\infty}\left|\frac{B_{t}(f)(x)-f(x)}{t^{\alpha}}-\frac{B_{t}(g)(x)}{t^{\alpha-2}}\right|^{2} \frac{d t}{t}\right)^{1 / 2},
\end{aligned}
$$

where $\alpha$ is a positive real number.

Theorem 1.1 ([1]). Let $p \in(1, \infty)$ and $\alpha \in(0,2]$. Then

(i) for $\alpha \in(0,2), f \in W^{\alpha, p}\left(\mathbb{R}^{n}\right)$ if and only if $f \in L^{p}\left(\mathbb{R}^{n}\right)$ and $T_{\alpha, 2}(f) \in L^{p}\left(\mathbb{R}^{n}\right)$;

(ii) $f \in W^{2, p}\left(\mathbb{R}^{n}\right)$ if and only if $f \in L^{p}\left(\mathbb{R}^{n}\right)$ and there exists a $g \in L^{p}\left(\mathbb{R}^{n}\right)$ such that $\mathcal{G}_{2,2}(f, g) \in L^{p}\left(\mathbb{R}^{n}\right)$.

As has already been pointed out in [5], Theorem 1.1 is actually a simple consequence of the following theorem. 
Theorem 1.2 ([9]). Let $\Phi$ be a nonzero integrable function. Suppose that for some $p_{0} \in(1, \infty)$,

$$
\text { (i) }\left\|\sup _{t>0}\left|\Phi_{t}\right| * f\right\|_{L^{q}\left(\mathbb{R}^{n}\right)} \preceq\|f\|_{L^{q}\left(\mathbb{R}^{n}\right)} \text { for } q>p_{0},
$$

and there exist $a, b>0$ such that for all $\xi \neq 0$;

$$
\text { (ii) }|\widehat{\Phi}(\xi)| \preceq \min \left\{|\xi|^{a},|\xi|^{-b}\right\} \text {. }
$$

Then

$$
\left\|S_{\Phi, 2}(f)\right\|_{L^{p}\left(\mathbb{R}^{n}\right)} \preceq\|f\|_{L^{p}\left(\mathbb{R}^{n}\right)}
$$

for all $\frac{2 p_{0}}{p_{0}+1}<p<\frac{2 p_{0}}{p_{0}-1}$.

Additionally, if $\Phi$ is a radial function. Then we have for $\frac{2 p_{0}}{p_{0}+1}<p<\frac{2 p_{0}}{p_{0}-1}$,

$$
\left\|S_{\Phi, 2}(f)\right\|_{L^{p}\left(\mathbb{R}^{n}\right)} \approx\|f\|_{L^{p}\left(\mathbb{R}^{n}\right)} .
$$

The main new idea of this work is to establish the sufficient condition for the boundedness of Littlewood-Paley functions $S_{\Phi, q}$ from homogeneous Triebel-Lizorkin spaces $\dot{F}_{p, q}^{0}\left(\mathbb{R}^{n}\right)$ (or homogeneous Besov spaces $\dot{B}_{p, q}^{0}\left(\mathbb{R}^{n}\right)$ ) to $L^{p}\left(\mathbb{R}^{n}\right)$ with $1<p, q<\infty$. These two theorems make the problem much simpler and also provide easier proofs of various previously known results. The rest of this paper is organized as follows. In Section 2, we will state the above two results and prove them. In the next three sections, we give some applications. In Section 3, we will characterize inhomogeneous Triebel-Lizorkin spaces $F_{p, q}^{\alpha}\left(\mathbb{R}^{n}\right)$ and inhomogeneous Besov spaces $B_{p, q}^{\alpha}\left(\mathbb{R}^{n}\right)$ for $0<\alpha<2$ by using several different square functions defined via the spherical average, the ball average and the Bochner-Riesz means. We also characterize homogeneous Triebel-Lizorkin spaces $\dot{F}_{p, q}^{\alpha}\left(\mathbb{R}^{n}\right)$ and homogeneous Besov spaces $\dot{B}_{p, q}^{\alpha}\left(\mathbb{R}^{n}\right)$ for $\alpha<0$ via the generalized BochnerRiesz means. In Section 4 we will characterize inhomogeneous Triebel-Lizorkin spaces $F_{p, q}^{m \alpha}\left(\mathbb{R}^{n}\right)$ and inhomogeneous Besov spaces $B_{p, q}^{m \alpha}\left(\mathbb{R}^{n}\right)$ for $0<\alpha<2$ and all $m \in \mathbb{Z}_{+}$via continuous and discrete versions of Littlewood-Paley functions. In the last section, we will use the Littlewood-Paley type function $S_{E, \alpha, q}$ to characterize inhomogeneous TriebelLizorkin spaces $F_{p, q}^{\alpha}\left(\mathbb{R}^{n}\right)$ and inhomogeneous Besov spaces $B_{p, q}^{\alpha}\left(\mathbb{R}^{n}\right)$. In fact, if $q=2$, the operator $S_{E, \alpha, q}$ is introduced by Sato et al. in [18] to characterize Sobolev spaces $W^{\alpha, p}\left(\mathbb{R}^{n}\right)$.

Throughout this article, the symbol $A \preceq B$ means that there exists a constant $C>0$ independent of all essential variables such that $A \leq C B$. We use the notation $A \approx B$ if $A \preceq B$ and $B \preceq A$. For $1<p<\infty$, let $p^{\prime}$ be the conjugate index of $p$, that is, $1 / p+1 / p^{\prime}=$ 1. If $p=1$, we define its conjugate index to be $p^{\prime}=\infty$.

\section{Characterize $\dot{F}_{p, q}^{0}\left(\mathbb{R}^{n}\right)$ and $\dot{B}_{p, q}^{0}\left(\mathbb{R}^{n}\right)$ using $S_{\Phi, q}$}

We recall some notation and notions. Denote by $C^{\infty}\left(\mathbb{R}^{n}\right)$ the set of infinitely differential functions on $\mathbb{R}^{n}$ and $C_{c}^{\infty}\left(\mathbb{R}^{n}\right)$ the set of $C^{\infty}\left(\mathbb{R}^{n}\right)$ functions with compact support. Let 
$\mathcal{S}_{\infty}\left(\mathbb{R}^{n}\right)$ be the set of functions $\phi \in \mathcal{S}\left(\mathbb{R}^{n}\right)$ satisfying

$$
\int_{\mathbb{R}^{n}} \phi(x) x^{\sigma} d x=0
$$

for all multi-indices $\sigma \in\left(\mathbb{Z}_{+}\right)^{n}$. Let $\mathcal{S}^{\prime}\left(\mathbb{R}^{n}\right)$ and $\mathcal{S}_{\infty}^{\prime}\left(\mathbb{R}^{n}\right)$ be the dual spaces of $\mathcal{S}\left(\mathbb{R}^{n}\right)$ and $\mathcal{S}_{\infty}\left(\mathbb{R}^{n}\right)$, respectively, and endow them with the weak-* topology. We use $\mathcal{A}$ to denote the class of all Schwartz functions $\phi$ on $\mathbb{R}^{n}$ such that supp $\hat{\phi} \subset\{1 / 2 \leq|\xi| \leq 2\}$ and $|\widehat{\phi}(\xi)| \geq C>0$ for $3 / 5 \leq|\xi| \leq 5 / 3$, where the Fourier transform $\widehat{\phi}$ is defined as

$$
\widehat{\phi}(\xi)=\int_{\mathbb{R}^{n}} \phi(x) e^{-2 \pi i \xi \cdot x} d x, \quad \xi \in \mathbb{R}^{n} .
$$

We set $\phi_{2^{-j}}(x)=2^{j n} \phi\left(2^{j} x\right)$ for $x \in \mathbb{R}^{n}$ and $j \in \mathbb{Z}$. Recall definitions of Triebel-Lizorkin and Besov spaces; see [12,23,24,27].

Definition 2.1. Assume that $\phi \in \mathcal{A}$. For $\alpha \in \mathbb{R}, p, q \in(0, \infty]$.

(i) The homogeneous Triebel-Lizorkin space $\dot{F}_{p, q}^{\alpha}\left(\mathbb{R}^{n}\right)$ is the collection of all $f \in \mathcal{S}_{\infty}^{\prime}\left(\mathbb{R}^{n}\right)$ such that $\|f\|_{\dot{F}_{p, q}^{\alpha}\left(\mathbb{R}^{n}\right)}<\infty$, where, when $p \in(0, \infty)$,

$$
\|f\|_{\dot{F}_{p, q}^{\alpha}\left(\mathbb{R}^{n}\right)}:=\left\|\left(\sum_{j \in \mathbb{Z}} 2^{j \alpha q}\left|\phi_{2^{-j}} * f\right|^{q}\right)^{1 / q}\right\|_{L^{p}\left(\mathbb{R}^{n}\right)} \approx\left\|\left(\int_{\mathbb{R}}\left(2^{-t \alpha}\left|\phi_{2^{t}} * f\right|\right)^{q} d t\right)^{1 / q}\right\|_{L^{p}\left(\mathbb{R}^{n}\right)}
$$

with the usual modification made when $q=\infty$, and

$$
\|f\|_{\dot{F}_{\infty, q}^{\alpha}\left(\mathbb{R}^{n}\right)}:=\sup _{x \in \mathbb{R}^{n}} \sup _{m \in \mathbb{Z}}\left\{\frac{1}{\left|B\left(x, 2^{-m}\right)\right|} \int_{B\left(x, 2^{-m}\right)} \sum_{j=m}^{\infty} 2^{j \alpha q}\left|\phi_{2^{-j}} * f(y)\right|^{q} d y\right\}^{1 / q}
$$

the usual modification made when $q=\infty$.

(ii) The homogeneous Besov space $\dot{B}_{p, q}^{\alpha}\left(\mathbb{R}^{n}\right)$ is the collection of all $f \in \mathcal{S}_{\infty}^{\prime}\left(\mathbb{R}^{n}\right)$ such that $\|f\|_{\dot{B}_{p, q}^{\alpha}\left(\mathbb{R}^{n}\right)}<\infty$, where

$$
\|f\|_{\dot{B}_{p, q}^{\alpha}\left(\mathbb{R}^{n}\right)}:=\left(\sum_{j \in \mathbb{Z}} 2^{j \alpha q}\left\|\phi_{2^{-j}} * f\right\|_{L^{p}\left(\mathbb{R}^{n}\right)}^{q}\right)^{1 / q} \approx\left(\int_{\mathbb{R}}\left(2^{-t \alpha}\left\|\phi_{2^{t}} * f\right\|_{L^{p}\left(\mathbb{R}^{n}\right)}\right)^{q} d t\right)^{1 / q}
$$

with the usual modification made when $p=\infty$ or $q=\infty$.

Note that $\dot{F}_{p, 2}^{0}\left(\mathbb{R}^{n}\right)=H^{p}\left(\mathbb{R}^{n}\right)$ for $0<p<\infty, \dot{F}_{\infty, 2}^{0}\left(\mathbb{R}^{n}\right)=B M O$, and the monotone imbedding property $\dot{F}_{p, q_{1}}^{\alpha}\left(\mathbb{R}^{n}\right) \hookrightarrow \dot{F}_{p, q_{2}}^{\alpha}\left(\mathbb{R}^{n}\right)$ for $0<q_{1} \leq q_{2} \leq \infty$ (see [11] or [14, Exe. 6.5.1, p. 76]). We also recall the known fact $H^{p}\left(\mathbb{R}^{n}\right)=L^{p}\left(\mathbb{R}^{n}\right)$ if $1<p<\infty$.

The corresponding inhomogeneous spaces are given as follows. 
Definition 2.2. Let $\alpha \in \mathbb{R}, p, q \in(1, \infty]$. Assume that $\phi \in \mathcal{A}$ and let $\Phi \in \mathcal{S}\left(\mathbb{R}^{n}\right)$ satisfy that

$$
\operatorname{supp} \widehat{\Phi} \subset\left\{\xi \in \mathbb{R}^{n}:|\xi| \leq 2\right\} \text { and }|\widehat{\Phi}(\xi)| \geq C>0 \text { if }|\xi| \leq 5 / 3 \text {. }
$$

(i) The inhomogeneous Triebel-Lizorkin space $F_{p, q}^{\alpha}\left(\mathbb{R}^{n}\right)$ is the collection of all $f \in \mathcal{S}^{\prime}\left(\mathbb{R}^{n}\right)$ such that such that $\|f\|_{F_{p, q}^{\alpha}\left(\mathbb{R}^{n}\right)}<\infty$, where, when $p \in(0, \infty)$,

$$
\|f\|_{F_{p, q}^{\alpha}\left(\mathbb{R}^{n}\right)}:=\left\|\left(\sum_{j \in \mathbb{Z}_{+}} 2^{j \alpha q}\left|\phi_{2^{-j}} * f\right|^{q}\right)^{1 / q}\right\|_{L^{p}\left(\mathbb{R}^{n}\right)}
$$

with the usual modification made when $q=\infty$, and

$$
\|f\|_{F_{\infty, q}^{\alpha}\left(\mathbb{R}^{n}\right)}:=\sup _{x \in \mathbb{R}^{n}} \sup _{m \in \mathbb{Z}_{+}}\left\{\frac{1}{\left|B\left(x, 2^{-m}\right)\right|} \int_{B\left(x, 2^{-m}\right)} \sum_{j=m}^{\infty} 2^{j \alpha q}\left|\phi_{2^{-j}} * f(y)\right|^{q} d y\right\}^{1 / q}
$$

with the usual modification made when $q=\infty$, where, when $j=0, \phi_{2^{-j}}$ is replaced by $\Phi$.

(ii) The inhomogeneous Besov space $B_{p, q}^{\alpha}\left(\mathbb{R}^{n}\right)$ is the collection of all $f \in \mathcal{S}_{\infty}^{\prime}\left(\mathbb{R}^{n}\right)$ such that $\|f\|_{B_{p, q}^{\alpha}\left(\mathbb{R}^{n}\right)}<\infty$, where

$$
\|f\|_{B_{p, q}^{\alpha}\left(\mathbb{R}^{n}\right)}:=\left(\sum_{j \in \mathbb{Z}_{+}} 2^{j \alpha q}\left\|\phi_{2^{-j}} * f\right\|_{L^{p}\left(\mathbb{R}^{n}\right)}^{q}\right)^{1 / q}
$$

with the usual modification made when $p=\infty$ or $q=\infty$, where, when $j=0, \phi_{2^{-j}}$ is replaced by $\Phi$.

From [17, Theorem 3.3.1] and [16, Chapter 2.2.4, Corollary 2], it is easy to see that

Definition 2.3. Let $\alpha>0, p, q \in(1, \infty]$. Assume that $\phi \in \mathcal{A}$ and let $\Phi \in \mathcal{S}\left(\mathbb{R}^{n}\right)$ satisfy that

$$
\text { supp } \widehat{\Phi} \subset\left\{\xi \in \mathbb{R}^{n}:|\xi| \leq 2\right\} \text { and }|\widehat{\Phi}(\xi)| \geq C>0 \text { if }|\xi| \leq 5 / 3 \text {. }
$$

(i) The inhomogeneous Triebel-Lizorkin space $F_{p, q}^{\alpha}\left(\mathbb{R}^{n}\right)$ is the collection of all $f \in \mathcal{S}^{\prime}\left(\mathbb{R}^{n}\right)$ such that such that $\|f\|_{F_{p, q}^{\alpha}\left(\mathbb{R}^{n}\right)}<\infty$, where

$$
\|f\|_{F_{p, q}^{\alpha}\left(\mathbb{R}^{n}\right)} \approx\|f\|_{L^{p}\left(\mathbb{R}^{n}\right)}+\|\widetilde{f}\|_{F_{p, q}^{\alpha}\left(\mathbb{R}^{n}\right)},
$$

where $\|\widetilde{f}\|_{F_{p, q}^{\alpha}\left(\mathbb{R}^{n}\right)}$ is defined as $\|f\|_{F_{p, q}^{\alpha}\left(\mathbb{R}^{n}\right)}$ in (i) of Definition 2.2 with $j \in \mathbb{Z}_{+}$and $m \in \mathbb{Z}_{+}$ therein replaced, respectively, by $j \in \mathbb{Z}$ and $m \in \mathbb{Z}$.

(ii) The inhomogeneous Besov space $B_{p, q}^{\alpha}\left(\mathbb{R}^{n}\right)$ is the collection of all $f \in \mathcal{S}_{\infty}^{\prime}\left(\mathbb{R}^{n}\right)$ such that $\|f\|_{B_{p, q}^{\alpha}\left(\mathbb{R}^{n}\right)}<\infty$, where

$$
\|f\|_{B_{p, q}^{\alpha}\left(\mathbb{R}^{n}\right)} \approx\|f\|_{L^{p}\left(\mathbb{R}^{n}\right)}+\|\widetilde{f}\|_{B_{p, q}^{\alpha}\left(\mathbb{R}^{n}\right)},
$$

where $\|\widetilde{f}\|_{B_{p, q}^{\alpha}\left(\mathbb{R}^{n}\right)}$ is defined as $\|f\|_{B_{p, q}^{\alpha}\left(\mathbb{R}^{n}\right)}$ in (ii) of Definition 2.2 with $j \in \mathbb{Z}_{+}$and $m \in$ $\mathbb{Z}_{+}$therein replaced, respectively, by $j \in \mathbb{Z}$ and $m \in \mathbb{Z}$. 
We firstly establish the following two theorems, which respectively are Triebel-Lizorkin analogy and Besov analogy of Theorem 1.2.

Theorem 2.1. Let $1<p, q<\infty$ and let $\Phi$ be a nonzero integrable function. Suppose that for some $p_{0} \in(1, \infty)$,

$$
\text { (i) } \quad\left\|\sup _{t>0}\left|\Phi_{t}\right| * f\right\|_{L^{q\left(\mathbb{R}^{n}\right)}} \preceq\|f\|_{L^{q}\left(\mathbb{R}^{n}\right)} \text { for } q>p_{0} ，
$$

and there exist $a, b>0$ such that for all $\xi \neq 0$;

$$
\text { (ii) }|\widehat{\Phi}(\xi)| \preceq \min \left\{|\xi|^{a},|\xi|^{-b}\right\} .
$$

Then

$$
\left\|S_{\Phi, q}(f)\right\|_{L^{p}\left(\mathbb{R}^{n}\right)} \preceq\|f\|_{\dot{F}_{p, q}^{0}\left(\mathbb{R}^{n}\right)}
$$

for all $\frac{2 p_{0}}{p_{0}+1}<p<\frac{2 p_{0}}{p_{0}-1}$.

Additionally, if $\Phi$ is a radial function. Then we have for $\frac{2 p_{0}}{p_{0}+1}<p<\frac{2 p_{0}}{p_{0}-1}$,

$$
\left\|S_{\Phi, q}(f)\right\|_{L^{p}\left(\mathbb{R}^{n}\right)} \approx\|f\|_{{\dot{\dot{P}_{p, q}^{0}}}_{\left(\mathbb{R}^{n}\right)}} .
$$

Proof. Without loss of generality, assume $a \geq b$. Take a radial function $\Psi \in \mathcal{S}\left(\mathbb{R}^{n}\right)$ satisfying the following conditions: (i) $0 \leq \widehat{\Psi}(\xi) \leq 1$, (ii) supp $\widehat{\Psi}(\xi) \subset\left\{\xi \in \mathbb{R}^{n}: 1 / 2 \leq|\xi| \leq\right.$ $2\}$, and (iii) $\int_{\mathbb{R}} \widehat{\Psi}\left(2^{s}\right)^{2} d s=1$. Then by the Calderón representation formula

$$
f=C \int_{\mathbb{R}}\left(\Psi_{2^{s}} * \Psi_{2^{s}} * f\right) d s
$$

for any $f$, where $C$ is a constant. Using the Minkowski inequality, we obtain that

$$
\begin{aligned}
S_{\Phi, q}(f)(x) & =\left(\int_{\mathbb{R}}\left|\Phi_{2^{t}} * f(x)\right|^{q} d t\right)^{1 / q} \\
& =C\left(\int_{\mathbb{R}}\left|\int_{\mathbb{R}} \Psi_{2^{s+t}} * \Psi_{2^{s+t}} * \Phi_{2^{t}} * f(x) d s\right|^{q} d t\right)^{1 / q} \\
& \preceq \int_{\mathbb{R}} \mathcal{T}_{s}(f)(x) d s,
\end{aligned}
$$

where

$$
\mathcal{T}_{s}(f)(x)=\left(\int_{\mathbb{R}}\left|\Psi_{2^{s+t}} * \Psi_{2^{s+t}} * \Phi_{2^{t}} * f(x)\right|^{q} d t\right)^{1 / q} .
$$

By the Minkowski inequality again, we have

$$
\left\|S_{\Phi, q}(f)\right\|_{L^{p}\left(\mathbb{R}^{n}\right)} \preceq \int_{\mathbb{R}}\left\|\mathcal{T}_{s}(f)\right\|_{L^{p}\left(\mathbb{R}^{n}\right)} d s .
$$


We will prove that there exists a constant $\sigma=\sigma(a, b, p, q)>0$ such that

$$
\left\|\mathcal{T}_{\mathcal{S}}(f)\right\|_{L^{p}\left(\mathbb{R}^{n}\right)} \preceq 2^{-\sigma|s|}\left\|S_{\Psi, q}(f)\right\|_{L^{p}\left(\mathbb{R}^{n}\right)},
$$

where

$$
S_{\Psi, q}(f)(x)=\left(\int_{\mathbb{R}}\left|\Psi_{2^{t}} * f(x)\right|^{q} d t\right)^{1 / q} .
$$

With this estimate, we have

$$
\left\|S_{\Phi, q}(f)\right\|_{L^{p}\left(\mathbb{R}^{n}\right)} \preceq\left\|S_{\Psi, q}\right\|_{L^{p}\left(\mathbb{R}^{n}\right)} \approx\|f\|_{\dot{F}_{p, q}^{0}\left(\mathbb{R}^{n}\right)} .
$$

Let $\mathcal{F}(y, t)=\Psi_{2^{s+t}} * f(y)$. Define a linear operator $T$ on functions $\mathcal{F}(y, t)$ by

$$
T(\mathcal{F})(x, t)=\Psi_{2^{s+t}} * \Phi_{2^{t}} * \mathcal{F}(x, t)=\int_{\mathbb{R}^{n}}\left(\Psi_{2^{s+t}} * \Phi_{2^{t}}\right)(x-y) \mathcal{F}(y, t) d y .
$$

By Fubini's theorem and the Young inequality, it is easy to check that for any $1 \leq r \leq \infty$, we have

$$
\begin{aligned}
\|\| T(\mathcal{F})\left\|_{L^{r}(\mathbb{R})}\right\|_{L^{r}\left(\mathbb{R}^{n}\right)}^{r} & =\int_{\mathbb{R}^{n}}\left(\int_{\mathbb{R}}\left|\Psi_{2^{s+t}} * \Phi_{2^{t}} * \mathcal{F}(x, t)\right|^{r} d t\right) d x \\
& \leq\|\Phi\|_{L^{1}}\|\Psi\|_{L^{1}} \int_{\mathbb{R}}\left(\int_{\mathbb{R}^{n}}|\mathcal{F}(x, t)|^{r} d x\right) d t \\
& \preceq\|\| \mathcal{F}\left\|_{L^{r}(\mathbb{R})}\right\|_{L^{r}\left(\mathbb{R}^{n}\right)}^{r} .
\end{aligned}
$$

It follows from the Young inequality and the condition (i) of Theorem 2.1, for any $r$, $1<r<\infty$,

$$
\|\| T(\mathcal{F})\left\|_{L^{\infty}(\mathbb{R})}\right\|_{L^{r}\left(\mathbb{R}^{n}\right)} \preceq\left\|\sup _{t \in \mathbb{R}}\left|\Phi_{2^{t}}\right| *|\mathcal{F}(\cdot, t)|\right\|_{L^{r}\left(\mathbb{R}^{n}\right)} \preceq\|\| \mathcal{F}\left\|_{L^{\infty}(\mathbb{R})}\right\|_{L^{r}\left(\mathbb{R}^{n}\right)} .
$$

Next we will estimate

$$
\|\| T(\mathcal{F})\left\|_{L^{2}(\mathbb{R})}\right\|_{L^{2}\left(\mathbb{R}^{n}\right)} \preceq 2^{-2 \min \{a, b\}|s|}\|\| \mathcal{F}\left\|_{L^{2}(\mathbb{R})}\right\|_{L^{2}\left(\mathbb{R}^{n}\right)} .
$$

We now divide $s$ into two cases: $s>0$ and $s \leq 0$.

If $s>0$, by the Fubini thorem and the Plancherel theorem, we have

$$
\begin{aligned}
\|\| T(\mathcal{F})\left\|_{L^{2}(\mathbb{R})}\right\|_{L^{2}\left(\mathbb{R}^{n}\right)} & =\int_{\mathbb{R}} \int_{\mathbb{R}^{n}}\left|(T(\mathcal{F}))^{\wedge}(\xi, t)\right|^{2} d \xi d t \\
& =\int_{\mathbb{R}} \int_{\mathbb{R}^{n}}\left|\widehat{\Psi}\left(2^{t+s} \xi\right) \widehat{\Phi}\left(2^{t} \xi\right) \widehat{F(\cdot, t)}(\xi)\right|^{2} d \xi d t \\
& \leq \int_{\mathbb{R}} \int_{E_{t+s}}\left|\widehat{\Phi}\left(2^{t} \xi\right) \widehat{F(\cdot, t)}(\xi)\right|^{2} d \xi d t,
\end{aligned}
$$


where

$$
E_{s+t}=\left\{\xi \in \mathbb{R}^{n}: 2^{-t-s-1} \leq|\xi| \leq 2^{-t-s+1}\right\} .
$$

It follows from the condition (ii) of the theorem and the Plancherel theorem that

$$
\begin{aligned}
\|\| T(\mathcal{F})\left\|_{L^{2}(\mathbb{R})}\right\|_{L^{2}\left(\mathbb{R}^{n}\right)} & \preceq \int_{\mathbb{R}} \int_{E_{t+s}}\left|2^{t} \xi\right|^{2 a}|\widehat{F(\cdot, t)}(\xi)|^{2} d \xi d t \\
& \preceq 2^{-2 s a} \int_{\mathbb{R}} \int_{E_{t+s}}|\widehat{F(\cdot, t)}(\xi)|^{2} d \xi d t \\
& \leq 2^{-2 s a}\|\| \mathcal{F}\left\|_{L^{2}(\mathbb{R})}\right\|_{L^{2}\left(\mathbb{R}^{n}\right)} .
\end{aligned}
$$

If $s \leq 0$, then we use the same argument as above to obtain

$$
\begin{aligned}
\|\| T(\mathcal{F})\left\|_{L^{2}(\mathbb{R})}\right\|_{L^{2}\left(\mathbb{R}^{n}\right)} & \preceq \int_{\mathbb{R}} \int_{E_{t+s}}\left|2^{t} \xi\right|^{-2 b}|\widehat{F(\cdot, t)}(\xi)|^{2} d \xi d t \\
& \preceq 2^{2 s b} \int_{\mathbb{R}} \int_{E_{t+s}}|\widehat{F(\cdot, t)}(\xi)|^{2} d \xi d t \\
& \leq 2^{2 s b}\|\| \mathcal{F}\left\|_{L^{2}(\mathbb{R})}\right\|_{L^{2}\left(\mathbb{R}^{n}\right)} .
\end{aligned}
$$

Thus we prove the estimate in (2.5).

Using an interpolation in [16, (i) of Proposition, p. 56] between $L^{2}\left(L^{2}\right)\left(\mathbb{R}, \mathbb{R}^{n}\right)$ and $L^{1}\left(L^{1}\right)\left(\mathbb{R}, \mathbb{R}^{n}\right)\left(\right.$ or $L^{\infty}\left(L^{\infty}\right)\left(\mathbb{R}, \mathbb{R}^{n}\right)$ ) for the operator $T(\mathcal{F})$, one has

$$
\|\| T(\mathcal{F})\left\|_{L^{p}(\mathbb{R})}\right\|_{L^{p}\left(\mathbb{R}^{n}\right)} \preceq 2^{-2|s| \sigma(a, b, p)}\|\| \mathcal{F}\left\|_{L^{p}(\mathbb{R})}\right\|_{L^{p}\left(\mathbb{R}^{n}\right)}
$$

for any $p \in(1, \infty)$, where $\sigma(p)$ is a positive constant depending on $a, b$ and $p$. Another interpolation between (2.4) and (2.5) yields that for all $1<p \leq q<\infty$, there is a positive constant $\delta(a, b, p, q)$ such that

$$
\|\| T(\mathcal{F})\left\|_{L^{q}(\mathbb{R})}\right\|_{L^{p}\left(\mathbb{R}^{n}\right)} \preceq 2^{-2|s| \sigma(a, b, p, q)}\|\| \mathcal{F}\left\|_{L^{q}(\mathbb{R})}\right\|_{L^{p}\left(\mathbb{R}^{n}\right)} .
$$

Finally a duality argument yields that the above inequality holds for all $p, q \in(1, \infty)$. In fact, for $1<q \leq p<\infty$, there exists a function $g(x, t)$ satisfying

$$
\|\| g\left\|_{L^{\prime}(\mathbb{R})}\right\|_{L^{p^{\prime}\left(\mathbb{R}^{n}\right)}}=1,
$$

such that

$$
\|\| T(\mathcal{F})\left\|_{L^{q}(\mathbb{R})}\right\|_{L^{p}\left(\mathbb{R}^{n}\right)}=\left|\int_{\mathbb{R}^{n}} \int_{\mathbb{R}} T(\mathcal{F})(x, t) g(x, t) d t d x\right| .
$$

Set

$$
T^{*}(g)(x, t)=\int_{\mathbb{R}^{n}}\left(\Psi_{2^{s+t}} * \Phi_{2^{t}}\right)(y-x) g(y, t) d y .
$$


It follows from Fubini's theorem that

$$
\begin{aligned}
\left\|\mathcal{T}_{s}(f)\right\|_{L^{p}\left(\mathbb{R}^{n}\right)} & =\|\| T(\mathcal{F})\left\|_{L^{q}(\mathbb{R})}\right\|_{L^{p}\left(\mathbb{R}^{n}\right)} \\
& =\left|\int_{\mathbb{R}^{n}} \int_{\mathbb{R}} \int_{\mathbb{R}^{n}}\left(\Psi_{2^{s+t}} * \Phi_{2^{t}}\right)(x-y) g(x, t) d x \mathcal{F}(y, t) d t d y\right| \\
& =\left|\int_{\mathbb{R}^{n}} \int_{\mathbb{R}} T^{*}(g)(y, t) \mathcal{F}(y, t) d t d y\right| \\
& \leq\|\| T^{*}(g)\left\|_{L^{q^{\prime}}(\mathbb{R})}\right\|_{L^{p^{\prime}}\left(\mathbb{R}^{n}\right)}\|\| \mathcal{F}\left\|_{L^{q}(\mathbb{R})}\right\|_{L^{p}\left(\mathbb{R}^{n}\right)} \\
& \preceq 2^{-2|s| \sigma(a, b, p, q)}\|\| \mathcal{F}\left\|_{L^{q}(\mathbb{R})}\right\|_{L^{p}\left(\mathbb{R}^{n}\right)} .
\end{aligned}
$$

For $1<q<\infty$, let $\widetilde{\Psi}(x)=\Psi(-x)$ and define

$$
S_{\widetilde{\Psi}, q}(f)(x)=\left(\int_{0}^{\infty}\left|\widetilde{\Psi}_{t} * f(x)\right|^{q} \frac{d t}{t}\right)^{1 / q},
$$

and define $S_{\widetilde{\Phi}, q}$ in the same way. Obviously,

$$
S_{\widetilde{\Phi}, q}(f)=S_{\Phi, q}(f),
$$

whenever $\Phi$ is a radial function. A similar argument as above shows that the following estimate

$$
\left\|S_{\widetilde{\Phi}, q}(f)\right\|_{L^{p}\left(\mathbb{R}^{n}\right)} \preceq\left\|S_{\widetilde{\Psi}, q}(f)\right\|_{L^{p}\left(\mathbb{R}^{n}\right)} \approx\|f\|_{\dot{F}_{p, q}^{0}\left(\mathbb{R}^{n}\right)}
$$

holds.

To show the reversed inequality

$$
\|f\|_{\dot{F}_{p, q}^{0}\left(\mathbb{R}^{n}\right)} \preceq\left\|S_{\Phi, q}(f)\right\|_{L^{p}\left(\mathbb{R}^{n}\right)},
$$

we will invoke the Calderón representation formula (2.1). We now claim that for any function $f_{t}(x)$,

$$
\left\|\int_{0}^{\infty}\left(\Phi_{t} * f_{t}\right)(\cdot) \frac{d t}{t}\right\|_{\dot{F}_{p, q}^{0}\left(\mathbb{R}^{n}\right)} \preceq\left\|\left(\int_{0}^{\infty}\left|f_{t}\right|^{q}(\cdot) \frac{d t}{t}\right)^{1 / q}\right\|_{L^{p}\left(\mathbb{R}^{n}\right)} .
$$

In fact, there is a $g \in \dot{F}_{p^{\prime}, q^{\prime}}^{0}$ with $\|g\|_{\dot{F}_{p^{\prime}, q^{\prime}}^{0}}=1$ such that

$$
\begin{aligned}
& \left.\left\|\int_{0}^{\infty}\left(\Phi_{t} * f_{t}\right)(\cdot) \frac{d t}{t}\right\|\right|_{\dot{F}_{, q,}^{0}\left(\mathbb{R}^{n}\right)} \\
= & \left|\left\langle\int_{0}^{\infty}\left(\Phi_{t} * f_{t}\right) \frac{d t}{t}, g\right\rangle\right|=\left|\int_{0}^{\infty}\left\langle\Phi_{t} * f_{t}, g\right\rangle \frac{d t}{t}\right|=\left|\int_{0}^{\infty}\left\langle f_{t}, \widetilde{\Phi}_{t} * g\right\rangle \frac{d t}{t}\right|,
\end{aligned}
$$


where

$$
\widetilde{\Phi}_{t} * g(x)=\int_{\mathbb{R}^{n}} \Phi_{t}(y-x) g(y) d y .
$$

By the Hölder inequality we now obtain that

$$
\begin{aligned}
\left|\int_{0}^{\infty}\left\langle f_{t}, \widetilde{\Phi}_{t} * g\right\rangle \frac{d t}{t}\right| & \preceq\left\|\left(\int_{0}^{\infty}\left|f_{t}\right|^{q}(\cdot) \frac{d t}{t}\right)^{1 / q}\right\|_{L^{p}\left(\mathbb{R}^{n}\right)}\left\|S_{\widetilde{\Phi}, q^{\prime}}(g)\right\|_{L^{p^{\prime}}\left(\mathbb{R}^{n}\right)} \\
& \preceq\left\|\left(\int_{0}^{\infty}\left|f_{t}\right|^{q}(\cdot) \frac{d t}{t}\right)^{1 / q}\right\|_{L^{p}\left(\mathbb{R}^{n}\right)}\|g\|_{\dot{F}_{p^{\prime}, q^{\prime}}\left(\mathbb{R}^{n}\right)} .
\end{aligned}
$$

Applying $f_{t}=\Phi_{t} * f$ to the above inequality in (2.6), we obtain that

$$
\begin{aligned}
\|f\|_{\dot{F}_{p, q}^{0}} & \approx\left\|\int_{0}^{\infty}\left(\Phi_{t} * \Phi_{t} * f\right)(\cdot) \frac{d t}{t}\right\|_{\dot{F}_{p, q}^{0}\left(\mathbb{R}^{n}\right)} \\
& \preceq\left\|\left(\int_{0}^{\infty}\left|\Phi_{t} * f\right|^{q}(\cdot) \frac{d t}{t}\right)^{1 / q}\right\|_{L^{p}\left(\mathbb{R}^{n}\right)} \\
& =\left\|S_{\Phi, q}(f)\right\|_{L^{p}\left(\mathbb{R}^{n}\right)},
\end{aligned}
$$

which completes the proof of the theorem.

Theorem 2.2. Let $1<p, q<\infty$ and let $\Phi$ be a nonzero integrable function. Suppose that there exist $a, b>0$ such that for all $\xi \neq 0$,

$$
|\widehat{\Phi}(\xi)| \preceq \min \left\{|\xi|^{a},|\xi|^{-b}\right\} .
$$

Then we have

$$
\left(\int_{\mathbb{R}}\left\|\Phi_{2^{t}} * f\right\|_{L^{p}\left(\mathbb{R}^{n}\right)}^{q} d t\right)^{1 / q} \preceq\|f\|_{\dot{B}_{p, q}^{0}\left(\mathbb{R}^{n}\right)} .
$$

Additionally, if $\Phi$ is a radial function. Then for $1<p, q<\infty$, we have

$$
\left(\int_{\mathbb{R}}\left\|\Phi_{2^{t}} * f\right\|_{L^{p}\left(\mathbb{R}^{n}\right)}^{q} d t\right)^{1 / q} \approx\|f\|_{\dot{B}_{p, q}^{0}\left(\mathbb{R}^{n}\right)} .
$$

Proof. Without loss of generality, assume $a \geq b$. Take a radial function $\Psi \in \mathcal{S}\left(\mathbb{R}^{n}\right)$ as in the proof of Theorem 2.1. As before, by the Calderón representation formula and the Minkowski inequality, we obtain that for $1<p, q<\infty$,

$$
\begin{aligned}
& \left(\int_{\mathbb{R}}\left\|\Phi_{2^{t}} * f\right\|_{L^{p}\left(\mathbb{R}^{n}\right)}^{q} d t\right)^{1 / q} \\
= & C\left(\int_{\mathbb{R}}\left\|\int_{\mathbb{R}} \Psi_{2^{s+t}} * \Psi_{2^{s+t}} * \Phi_{2^{t}} * f(x) d s\right\|_{L^{p}\left(\mathbb{R}^{n}\right)}^{q} d t\right)^{1 / q} \\
\preceq & \int_{\mathbb{R}} \mathcal{T}(f)(s) d s,
\end{aligned}
$$


where

$$
\mathcal{T}(f)(s)=\left(\int_{\mathbb{R}}\left\|\Psi_{2^{s+t}} * \Psi_{2^{s+t}} * \Phi_{2^{t}} * f\right\|_{L^{p}\left(\mathbb{R}^{n}\right)}^{q} d t\right)^{1 / q} .
$$

We will prove that there exists a constant $\sigma=\sigma(a, b, p, q)>0$ such that for $1<p, q<\infty$

$$
\mathcal{T}(f)(s) \preceq 2^{-\sigma|s|}\|f\|_{\dot{B}_{p, q}^{0}\left(\mathbb{R}^{n}\right)} .
$$

If it is so, then we have

$$
\left(\int_{\mathbb{R}}\left\|\Phi_{2^{t}} * f\right\|_{L^{p}\left(\mathbb{R}^{n}\right)}^{q} d t\right)^{1 / q} \preceq\|f\|_{\dot{B}_{p, q}^{0}\left(\mathbb{R}^{n}\right)} \int_{\mathbb{R}} 2^{-\sigma|s|} d s \preceq\|f\|_{\dot{B}_{p, q}^{0}\left(\mathbb{R}^{n}\right)} .
$$

We now prove (2.7). Define a linear operator $T$ on functions $\mathcal{F}(y, t)$ by

$$
T(\mathcal{F})(x, t)=\Psi_{2^{s+t}} * \Phi_{2^{t}} * \mathcal{F}(x, t)=\int_{\mathbb{R}^{n}}\left(\Psi_{2^{s+t}} * \Phi_{2^{t}}\right)(x-y) \mathcal{F}(y, t) d y,
$$

where $\mathcal{F}(y, t)=\Psi_{2^{s+t}} * f(y)$. As in the proof of Theorem 2.1, we can obtain that for all $1<p \leq q<\infty$, there is a positive constant $\delta(a, b, p, q)$ such that

$$
\|\| T(\mathcal{F})\left\|_{L^{p}\left(\mathbb{R}^{n}\right)}\right\|_{L^{q}(\mathbb{R})} \preceq 2^{-2|s| \sigma(a, b, p, q)}\|\| \mathcal{F}\left\|_{L^{p}\left(\mathbb{R}^{n}\right)}\right\|_{L^{q}(\mathbb{R})} .
$$

Thus the inequality (2.7) holds by taking $\mathcal{F}(y, t)=\Psi_{2^{s+t}} * f(y)$ in (2.8).

Finally a duality argument yields that the above inequality in (2.8) holds for all $p, q \in$ $(1, \infty)$. In fact, for $1<q \leq p<\infty$, there exists a function $\mathcal{H}(x, t)$ satisfying

$$
\|\| \mathcal{H}\left\|_{L^{p^{\prime}}\left(\mathbb{R}^{n}\right)}\right\|_{L^{q^{\prime}}(\mathbb{R})}=1
$$

such that

$$
\|\| T(\mathcal{F})\left\|_{L^{p}\left(\mathbb{R}^{n}\right)}\right\|_{L^{q}(\mathbb{R})}=\left|\int_{\mathbb{R}^{n}} \int_{\mathbb{R}} T(\mathcal{F})(x, t) \mathcal{H}(x, t) d t d x\right| .
$$

Let

$$
T^{*}(\mathcal{H})(x, t)=\int_{\mathbb{R}^{n}}\left(\Psi_{2^{s+t}} * \Phi_{2^{t}}\right)(y-x) \mathcal{H}(y, t) d y
$$

Since $\Psi$ is radial, we see that $T^{*}(\mathcal{H})(x, t)=T(\mathcal{H})(x, t)$ for all $(x, t) \in \mathbb{R}^{n} \times \mathbb{R}$. It follows 
from Fubini's theorem and (2.8) that

$$
\begin{aligned}
\mathcal{T}(f)(s) & =\|\| T(\mathcal{F})\left\|_{L^{p}\left(\mathbb{R}^{n}\right)}\right\|_{L^{q}(\mathbb{R})} \\
& =\left|\int_{\mathbb{R}^{n}} \int_{\mathbb{R}} \int_{\mathbb{R}^{n}}\left(\Psi_{2^{s+t}} * \Phi_{2^{t}}\right)(x-y) \mathcal{H}(x, t) d x \mathcal{F}(y, t) d t d y\right| \\
& =\left|\int_{\mathbb{R}} \int_{\mathbb{R}^{n}} T^{*}(\mathcal{H})(y, t) \mathcal{F}(y, t) d y d t\right| \\
& \leq\|\| T^{*}(\mathcal{H})\left\|_{L^{p^{\prime}}\left(\mathbb{R}^{n}\right)}\right\|_{L^{q^{\prime}}(\mathbb{R})}\|\| \mathcal{F}\left\|_{L^{p}\left(\mathbb{R}^{n}\right)}\right\|_{L^{q}(\mathbb{R})} \\
& \preceq 2^{-2|s| \sigma(a, b, p, q)}\|\| \mathcal{F}\left\|_{L^{p}\left(\mathbb{R}^{n}\right)}\right\|_{L^{q}(\mathbb{R})} \\
& \approx C 2^{-2|s| \sigma(a, b, p, q)}\|f\|_{\dot{B}_{p, q}^{0}\left(\mathbb{R}^{n}\right)} .
\end{aligned}
$$

Assume that $\Phi$ is a radial function. We will show that for $1<p, q<\infty$,

$$
\|f\|_{\dot{B}_{p, q}^{0}\left(\mathbb{R}^{n}\right)} \preceq\left(\int_{\mathbb{R}}\left\|\Phi_{2^{t}} * f\right\|_{L^{p}\left(\mathbb{R}^{n}\right)}^{q} d t\right)^{1 / q} .
$$

By the Calderón representation formula, we claim that for any function $f_{t}(x)$,

$$
\left\|\int_{0}^{\infty}\left(\Phi_{t} * f_{t}\right)(\cdot) \frac{d t}{t}\right\|_{\dot{B}_{p, q}^{0}\left(\mathbb{R}^{n}\right)} \preceq\left(\int_{0}^{\infty}\left\|\left|f_{t}\right|(\cdot)\right\|_{L^{p}\left(\mathbb{R}^{n}\right)}^{q} \frac{d t}{t}\right)^{1 / q} .
$$

In fact, there is a $g \in \dot{B}_{p^{\prime}, q^{\prime}\left(\mathbb{R}^{n}\right)}^{0}$ with $\|g\|_{\dot{B}_{p^{\prime}, q^{\prime}}^{0}\left(\mathbb{R}^{n}\right)}=1$ such that

$$
\begin{aligned}
& \left\|\int_{0}^{\infty}\left(\Phi_{t} * f_{t}\right)(\cdot) \frac{d t}{t}\right\|_{\dot{B}_{p, q}^{0}\left(\mathbb{R}^{n}\right)}=\left|\left\langle\int_{0}^{\infty}\left(\Phi_{t} * f_{t}\right) \frac{d t}{t}, g\right\rangle\right| \\
= & \left|\int_{0}^{\infty}\left\langle\Phi_{t} * f_{t}, g\right\rangle \frac{d t}{t}\right|=\left|\int_{0}^{\infty}\left\langle f_{t}, \widetilde{\Phi}_{t} * g\right\rangle \frac{d t}{t}\right|,
\end{aligned}
$$

where

$$
\widetilde{\Phi}_{t} * g(x)=\int_{\mathbb{R}^{n}} \Phi_{t}(y-x) g(y) d y .
$$

By the Hölder inequality, we now obtain that

$$
\begin{aligned}
\left|\int_{0}^{\infty}\left\langle f_{t}, \widetilde{\Phi}_{t} * g\right\rangle \frac{d t}{t}\right| & \preceq\left|\int_{0}^{\infty}\left\|f_{t}\right\|_{L^{p}\left(\mathbb{R}^{n}\right)}\left\|\Phi_{t} * g\right\|_{L^{p^{\prime}}\left(\mathbb{R}^{n}\right)} \frac{d t}{t}\right| \\
& \preceq\left(\int_{0}^{\infty}\left\|\left|f_{t}\right|(\cdot)\right\|_{L^{p}\left(\mathbb{R}^{n}\right)}^{q} \frac{d t}{t}\right)^{1 / q}\|g\|_{\dot{B}_{p^{\prime}, q^{\prime}}^{0}\left(\mathbb{R}^{n}\right)} .
\end{aligned}
$$


Applying $f_{t}=\Phi_{t} * f$ to the above inequality in (2.9), we obtain that

$$
\begin{aligned}
\|f\|_{\dot{B}_{p, q}^{0}\left(\mathbb{R}^{n}\right)} & \approx\left\|\int_{0}^{\infty}\left(\Phi_{t} * \Phi_{t} * f\right)(\cdot) \frac{d t}{t}\right\|_{\dot{B}_{p, q}^{0}\left(\mathbb{R}^{n}\right)} \\
& \preceq\left(\int_{0}^{\infty}\left\|\Phi_{t} * f\right\|_{L^{p}\left(\mathbb{R}^{n}\right)}^{q} \frac{d t}{t}\right)^{1 / q},
\end{aligned}
$$

which finishes the proof of the theorem.

\section{Characterizations of $F_{p, q}^{\alpha}$ and $B_{p, q}^{\alpha}$ for $0<\alpha<2$, and $\dot{F}_{p, q}^{\alpha}$ and $\dot{B}_{p, q}^{\alpha}$ for $\alpha<0$}

In this section, as applications of Theorems 2.1 and 2.2, we will consider two classes of Littlewood-Paley functions to characterize Triebel-Lizorkin and Besov spaces, respectively.

For $\alpha>0$ and $1<q<\infty$, the Littlewood-Paley function $S_{\Phi, \alpha, q}(f)$ is defined by

$$
S_{\Phi, \alpha, q}(f)(x)=\left(\int_{0}^{\infty}\left|\Phi_{t} * f(x)-f(x)\right|^{q} \frac{d t}{t^{1+\alpha q}}\right)^{1 / q},
$$

where $\Phi$ are integrable functions. By the Fourier transform, we may write

$$
S_{\Phi, \alpha, q}(f)(x) \approx S_{\Psi, q}\left((-\Delta)^{\alpha / 2}(f)\right)(x),
$$

where

$$
\widehat{\Psi}(\xi)=\frac{\widehat{\Phi}(\xi)-1}{(2 \pi|\xi|)^{\alpha}} .
$$

We introduce the following simple form of Theorems 2.1 and 2.2, which is suitable for these Littlewood-Paley type functions.

Proposition 3.1. Let $\alpha>0$ and $1<q<\infty$. Suppose that for some $p_{0} \in(1, \infty)$. Assume that $\Phi$ is a radial function and let

$$
\widehat{\Psi}(\xi)=\frac{\widehat{\Phi}(\xi)-1}{(2 \pi|\xi|)^{\alpha}} .
$$

If $\Psi$ is a nonzero integrable function and

$$
|\widehat{\Psi}(\xi)| \preceq \min \left\{|\xi|^{a},|\xi|^{-b}\right\}
$$

for some positive numbers $a$ and $b$. Then we have the following results:

$$
\text { (i) } \quad\left\|S_{\Phi, \alpha, q}(f)\right\|_{L^{p}\left(\mathbb{R}^{n}\right)}+\|f\|_{L^{p}\left(\mathbb{R}^{n}\right)} \approx\|f\|_{B_{p, q}^{\alpha}\left(\mathbb{R}^{n}\right)}
$$


provided $\frac{2 p_{0}}{p_{0}+1}<p<\frac{2 p_{0}}{p_{0}-1}$

(ii) If $\Psi$ also satisfies that for $p_{0}<p<\infty$,

$$
\left\|\sup _{t>0}\left|\Psi_{t}\right| * f\right\|_{L^{p}\left(\mathbb{R}^{n}\right)} \preceq\|f\|_{L^{p}\left(\mathbb{R}^{n}\right)},
$$

then we have that

$$
\left\|S_{\Phi, \alpha, q}(f)\right\|_{L^{p}\left(\mathbb{R}^{n}\right)}+\|f\|_{L^{p}\left(\mathbb{R}^{n}\right)} \approx\|f\|_{F_{p, q}^{\alpha}\left(\mathbb{R}^{n}\right)}
$$

provided $\frac{2 p_{0}}{p_{0}+1}<p<\frac{2 p_{0}}{p_{0}-1}$.

As an application of Proposition 3.1, we will study the following Littlewood-Paley function

$$
S_{V, \alpha, q}(f)(x)=\left(\int_{0}^{\infty}\left|V_{t} * f(x)-f(x)\right|^{q} \frac{d t}{t^{1+\alpha q}}\right)^{1 / q}
$$

for $1<q<\infty$ and $\alpha>0$. Here, $V_{t}$ is the spherical average (see [21]) given by

$$
V_{t}(f)(x)=\int_{\mathrm{S}^{n-1}} f(x-t y) d \sigma(y)
$$

and $\mathbb{S}^{n-1}$ is the unit sphere in $\mathbb{R}^{n}$ with the induced Lebesgue measure $d \sigma(y)$. Its corresponding maximal function is defined by

$$
\mathcal{V}(f)(x)=\sup _{t>0}\left|V_{t}(f)(x)\right| \text {. }
$$

The following result was obtained by Stein [21, Theorem 1, p. 2174], in dimensions $n \geq 3$, and Bourgain [2, Theorem 1, p. 69] in the delicate case of $n=2$.

Lemma 3.1. Suppose $n \geq 2$. Then for $f \in L^{p}\left(\mathbb{R}^{n}\right)$, the inequality

$$
\|\mathcal{V}(f)\|_{L^{p}\left(\mathbb{R}^{n}\right)} \leq C\|f\|_{L^{p}\left(\mathbb{R}^{n}\right)}
$$

holds whenever $n /(n-1)<p \leq \infty$. If $p \leq n /(n-1)$, then the inequality is not valid.

This lemma together with Corollaries 2.1-2.3 in [5] leads to the following results.

Corollary 3.1. Let $n \geq 2$ and $1<q<\infty$. For the spherical average $S_{V, \alpha, q}(f)$, if $0<\alpha \leq 1$, then we have that

$$
\left\|S_{V, \alpha, q}(f)\right\|_{L^{p}\left(\mathbb{R}^{n}\right)}+\|f\|_{L^{p}\left(\mathbb{R}^{n}\right)} \approx\|f\|_{F_{p, q}^{\alpha}\left(\mathbb{R}^{n}\right)}
$$

provided $\frac{2 n}{2 n-1}<p<2 n$. If $1<\alpha<2$ then we have that for all $p \in(1, \infty)$,

$$
\left\|S_{V, \alpha, q}(f)\right\|_{L^{p}\left(\mathbb{R}^{n}\right)}+\|f\|_{L^{p}\left(\mathbb{R}^{n}\right)} \approx\|f\|_{F_{p, q}^{\alpha}\left(\mathbb{R}^{n}\right)} .
$$


Corollary 3.2. Let $n \geq 2$ and $1<q<\infty$. For the spherical average $S_{V, \alpha, q}(f)$, if $0<\alpha \leq 1$, then we have that

$$
\left\|S_{V, \alpha, q}(f)\right\|_{L^{p}\left(\mathbb{R}^{n}\right)}+\|f\|_{L^{p}\left(\mathbb{R}^{n}\right)} \approx\|f\|_{B_{p, q}^{\alpha}\left(\mathbb{R}^{n}\right)}
$$

provided $\frac{2 n}{2 n-1}<p<2 n$. If $1<\alpha<2$, then we have that for all $p \in(1, \infty)$,

$$
\left\|S_{V, \alpha, q}(f)\right\|_{L^{p}\left(\mathbb{R}^{n}\right)}+\|f\|_{L^{p}\left(\mathbb{R}^{n}\right)} \approx\|f\|_{B_{p, q}^{\alpha}\left(\mathbb{R}^{n}\right)} .
$$

Corollary 3.3. Let $0<\alpha<2$ and $1<q<\infty$, and let $\Phi_{t} * f=B_{t}(f)$ be the ball average and

$$
S_{B, \alpha, q}(f)(x)=\left(\int_{0}^{\infty}\left|B_{t} f(x)-f(x)\right|^{q} \frac{d t}{t^{1+\alpha q}}\right)^{1 / q} .
$$

Then

$$
\begin{aligned}
& \left\|S_{B, \alpha, q}(f)\right\|_{L^{p}\left(\mathbb{R}^{n}\right)}+\|f\|_{L^{p}\left(\mathbb{R}^{n}\right)} \approx\|f\|_{F_{p, q}^{\alpha}\left(\mathbb{R}^{n}\right)}, \\
& \left\|S_{B, \alpha, q}(f)\right\|_{L^{p}\left(\mathbb{R}^{n}\right)}+\|f\|_{L^{p}\left(\mathbb{R}^{n}\right)} \approx\|f\|_{B_{p, q}^{\alpha}\left(\mathbb{R}^{n}\right)},
\end{aligned}
$$

for all $1<p<\infty$.

Let $t>0$. The generalized Bochner-Riesz means $B_{t}^{\delta, \gamma}$ on $\mathbb{R}^{n}$ are defined via the Fourier transform by

$$
\left(B_{t}^{\delta, \gamma}(f)\right)^{\wedge}(\xi)=\left(1-\frac{|\xi|^{\gamma}}{t^{\gamma}}\right)_{+}^{\delta} \widehat{f}(\xi)
$$

where $\delta$ and $\gamma$ are two real numbers satisfying $\delta>-1$ and $\gamma>0$. Again, we initially assume that $f$ are functions in $\mathcal{S}\left(\mathbb{R}^{n}\right)$. Use Proposition 3.1 to obtain the following result.

Corollary 3.4. Let $\alpha>0, \gamma>0,1<q<\infty$, and let $S_{B^{\delta, \gamma}, \alpha, q}$ be the operator

$$
\begin{aligned}
S_{B^{\delta, \gamma, \alpha, q}}(f)(x) & =\left(\int_{0}^{\infty}\left|B_{t}^{\delta, \gamma}(f)(x)-f(x)\right|^{q} \frac{d t}{t^{1+\alpha q}}\right)^{1 / q} \\
& \approx S_{\Psi, q}\left((-\Delta)^{\alpha / 2}(f)\right)(x),
\end{aligned}
$$

where

$$
\widehat{\Psi}(\xi)=\frac{\left(1-|\xi|^{\gamma}\right)_{+}^{\delta}-1}{(2 \pi|\xi|)^{\alpha}}
$$

Then for $\delta>\frac{n-1}{2}$ and $0<\alpha<\gamma$,

$$
\begin{aligned}
& \left\|S_{B^{\delta, \gamma}, \alpha, q}(f)\right\|_{L^{p}\left(\mathbb{R}^{n}\right)}+\|f\|_{L^{p}\left(\mathbb{R}^{n}\right)} \approx\|f\|_{F_{p, q}^{\alpha}\left(\mathbb{R}^{n}\right)}, \\
& \left\|S_{B^{\delta, \gamma}, \alpha, q}(f)\right\|_{L^{p}\left(\mathbb{R}^{n}\right)}+\|f\|_{L^{p}\left(\mathbb{R}^{n}\right)} \approx\|f\|_{B_{p, q}^{\alpha}\left(\mathbb{R}^{n}\right)},
\end{aligned}
$$

for all $1<p<\infty$. 
Next, we turn to characterize the homogeneous Triebel-Lizorkin space $\dot{F}_{p, q}^{-\alpha}$ and the homogeneous Besov space $\dot{B}_{p, q}^{-\alpha}$ for $\alpha>0$. To this end, we consider square functions of the form

$$
\mathbf{S}_{\Phi,-\alpha, q}(f)(x)=\left(\int_{0}^{\infty}\left|\Phi_{t} * f(x)\right|^{q} t^{\alpha q} \frac{d t}{t}\right)^{1 / q} .
$$

Again, we introduce the following simple form of Theorems 2.1 and 2.2 which is suitable for the above square function.

Proposition 3.2. Let $\alpha>0$ and $1<p, q<\infty$. Assume that $\Phi$ is a radial function and let

$$
\widehat{K}(\xi)=\widehat{\Phi}(\xi)(2 \pi|\xi|)^{\alpha} .
$$

If $K$ is a nonzero integrable function, and suppose that $a$ and $b$ are two positive numbers such that

$$
|\widehat{K}(\xi)| \preceq \min \left\{|\xi|^{a},|\xi|^{-b}\right\},
$$

then we have

$$
\text { (i) } \quad\left\|\mathbf{S}_{\Phi,-\alpha, q}(f)\right\|_{L^{p}\left(\mathbb{R}^{n}\right)} \approx\|f\|_{\dot{B}_{p, q}^{-\alpha}\left(\mathbb{R}^{n}\right)} .
$$

(ii) if K also satisfies that

$$
\left\|\sup _{t>0}\left|K_{t}\right| * f\right\|_{L^{p}\left(\mathbb{R}^{n}\right)} \preceq\|f\|_{L^{p}\left(\mathbb{R}^{n}\right)},
$$

then

$$
\left\|\mathbf{S}_{\Phi,-\alpha}(f)\right\|_{L^{p}\left(\mathbb{R}^{n}\right)} \approx\|f\|_{\dot{F}_{p, q}^{-\alpha}\left(\mathbb{R}^{n}\right)} .
$$

In order to use Proposition 3.2, we study a family of operators $\Omega_{t}^{\gamma}$ (see [21]) that takes the spherical mean $V_{t}$ and ball average $B_{t}$ as special cases. For $\gamma>0$, define

$$
\Omega_{t}^{\gamma}(f)(x)=C_{n, \gamma} t^{-n} \int_{|y|<t}\left(1-\left|\frac{y}{t}\right|^{2}\right)^{\gamma-1} f(x-y) d y,
$$

where

$$
C_{n, \gamma}=\frac{\Gamma(\gamma+n / 2)}{\pi^{n / 2} \Gamma(\gamma)}
$$

When $\gamma=1, \Omega_{t}^{1}(f)$ is the ball average

$$
\Omega_{t}^{1}(f)(x)=B_{t}(f)(x)=\frac{1}{|B(x, t)|} \int_{B(x, t)} f(y) d y .
$$

Also, by taking the Fourier transform, one can embed these operators in an analytic family of operators with complex parameter $\gamma$ so that we obtain the spherical average

$$
\Omega_{t}^{0}(f)(x)=V_{t}(f)(x) .
$$

With the help of Corollary 2.4 in [5, p. 284], we have 
Corollary 3.5. For two positive numbers $\gamma$ and $\alpha$, let

$$
\Phi_{t} * f(x)=\Omega_{t}^{\gamma}(f)(x)
$$

and for $1<q<\infty$

$$
\mathbf{S}_{\Omega^{\gamma},-\alpha, q}(f)(x)=\left(\int_{0}^{\infty}\left|\Omega_{t}^{\gamma}(f)(x)\right|^{q} \frac{t^{\alpha q} d t}{t}\right)^{1 / q}
$$

Then for $\gamma>\alpha+1$ and $1<p<\infty$

$$
\begin{aligned}
& \left\|\mathbf{S}_{\Omega^{\gamma,-\alpha, q}}(f)\right\|_{L^{p}\left(\mathbb{R}^{n}\right)} \approx\|f\|_{\dot{F}_{,, q}^{-\alpha}\left(\mathbb{R}^{n}\right)}, \\
& \left\|\mathbf{S}_{\Omega^{\gamma,-\alpha, q}}(f)\right\|_{L^{p}\left(\mathbb{R}^{n}\right)} \approx\|f\|_{\dot{B}_{p, q}^{-\alpha}\left(\mathbb{R}^{n}\right)} .
\end{aligned}
$$

\section{Characterizations of $F_{p, q}^{m \alpha}$ and $B_{p, q}^{m \alpha}$ for $0<\alpha<4$ and $m \in \mathbb{Z}_{+}$}

In this section, we assume that $1<q<\infty$ and $m \in \mathbb{Z}_{+}$. We will firstly study LittlewoodPaley functions as follows

$$
T_{\alpha, m, q}(f)(x)=\left(\int_{0}^{\infty}\left|\frac{\left(B_{t}-I\right)^{m}(f)(x)}{t^{m \alpha}}\right|^{q} \frac{d t}{t}\right)^{1 / q},
$$

and

$$
\mathcal{G}_{\alpha, m, c, q}(f)(x)=\left(\int_{0}^{\infty}\left|\frac{\left(\left(B_{t}-I\right)-c t^{q} B_{t} \circ(-\Delta)\right)^{m}(f)(x)}{t^{m \alpha}}\right|^{q} \frac{d t}{t}\right)^{1 / q},
$$

where $I$ is the identity operator, and $c$ is a constant. Note $T_{\alpha, 1, q}=T_{q, \alpha}$ and $\mathcal{G}_{\alpha, 1, c, q}(f)=$ $\mathcal{G}_{q, \alpha}(f, g)$, if $g=-c \Delta(f)$.

With the help of results in [5, Theorems 3.1 and 3.2, p. 285], we have the following statements.

Theorem 4.1. Let $p, q \in(1, \infty)$ and $\alpha \in(0,2)$. Then $f \in F_{p, q}^{m \alpha}\left(\mathbb{R}^{n}\right)$ if and only if $f \in L^{p}\left(\mathbb{R}^{n}\right)$ and $T_{\alpha, m, q}(f) \in L^{p}\left(\mathbb{R}^{n}\right)$.

Theorem 4.2. Let $p, q \in(1, \infty)$ and $\alpha \in(0,2)$. Then $f \in B_{p, q}^{m \alpha}\left(\mathbb{R}^{n}\right)$ if and only if $f \in L^{p}\left(\mathbb{R}^{n}\right)$ and $T_{\alpha, m, q}(f) \in L^{p}\left(\mathbb{R}^{n}\right)$.

Theorem 4.3. Let $p, q \in(1, \infty)$ and $\alpha \in[2,4)$. Then $f \in F_{p, q}^{m \alpha}\left(\mathbb{R}^{n}\right)$ if and only if $f \in L^{p}\left(\mathbb{R}^{n}\right)$ and $\mathcal{G}_{\alpha, m, c, q}(f) \in L^{p}\left(\mathbb{R}^{n}\right)$, where $c=-\frac{2 \pi^{2}}{(2 \pi)^{\alpha}(n+2)}$.

Theorem 4.4. Let $p, q \in(1, \infty)$ and $\alpha \in[2,4)$. Then $f \in B_{p, q}^{m \alpha}\left(\mathbb{R}^{n}\right)$ if and only if $f \in L^{p}\left(\mathbb{R}^{n}\right)$ and $\mathcal{G}_{\alpha, m, c, q}(f) \in L^{p}\left(\mathbb{R}^{n}\right)$, where $c=-\frac{2 \pi^{2}}{(2 \pi)^{\alpha}(n+2)}$. 
Next we will define the discrete versions of $T_{\alpha, m, q}$ and $\mathcal{G}_{\alpha, m, c, q}(f)$ by

$$
\begin{aligned}
& \mathcal{D}_{\alpha, m, q}(f)(x)=\left(\sum_{k \in \mathbb{Z}}\left|2^{-m \alpha k}\left(B_{2^{k}}-I\right)^{m}(f)(x)\right|^{q}\right)^{1 / q}, \\
& \mathcal{D}_{\alpha, m, c, q}(f)(x)=\left(\sum_{k \in \mathbb{Z}}\left|2^{-m \alpha k}\left(\left(B_{2^{k}}-I\right)-c 2^{2 k}\left(B_{2^{k}} \circ(-\Delta)\right)\right)^{m}(f)(x)\right|^{q}\right)^{1 / q} .
\end{aligned}
$$

Similar as proofs of Theorems 2.1 and 2.2, we have

Proposition 4.1. Let $1<p, q<\infty$. Suppose that $\Phi \in L^{1}$ is a radial function. Assume that there exist $a, b>0$ such that

$$
|\widehat{\Phi}(\xi)| \preceq \min \left\{|\xi|^{a},|\xi|^{-b}\right\} \text {. }
$$

Then we have

$$
\text { (i) }\left(\int_{\mathbb{R}}\left\|\Phi_{2^{t}} * f\right\|_{L^{p}\left(\mathbb{R}^{n}\right)}^{q} d t\right)^{1 / q} \approx\|f\|_{\dot{B}_{p, q}^{0}\left(\mathbb{R}^{n}\right)} .
$$

(ii) If $\Phi$ also satisfies that

$$
\left\|\sup _{k \in \mathbb{Z}}\left|\Phi_{2^{k}}\right| * f\right\|_{L^{p}\left(\mathbb{R}^{n}\right)} \preceq\|f\|_{L^{p}\left(\mathbb{R}^{n}\right)} \quad \text { for all } p>1,
$$

then for the operator $D_{\Phi, q}$ defined in (1.1), we have

$$
\left\|D_{\Phi, q}(f)\right\|_{L^{p}\left(\mathbb{R}^{n}\right)} \approx\|f\|_{\dot{F}_{p, q}^{0}\left(\mathbb{R}^{n}\right)}
$$

for all $1<p<\infty$.

By Proposition 4.1 and the results due to Chen et al. [5, Theorems 5.1 and 5.2, p. 293], we have the following results.

Theorem 4.5. Let $p, q \in(1, \infty)$ and $\alpha \in(0,2)$. Then $f \in F_{p, q}^{m \alpha}\left(\mathbb{R}^{n}\right)$ if and only if $f \in L^{p}\left(\mathbb{R}^{n}\right)$ and $\mathcal{D}_{\alpha, m, q}(f) \in L^{p}\left(\mathbb{R}^{n}\right)$.

Theorem 4.6. Let $p, q \in(1, \infty)$ and $\alpha \in(0,2)$. Then $f \in B_{p, q}^{m \alpha}\left(\mathbb{R}^{n}\right)$ if and only if $f \in L^{p}\left(\mathbb{R}^{n}\right)$ and $\mathcal{D}_{\alpha, m, q}(f) \in L^{p}\left(\mathbb{R}^{n}\right)$.

Theorem 4.7. Let $p, q \in(1, \infty)$ and $\alpha \in[2,4)$. Then $f \in F_{p, q}^{m \alpha}\left(\mathbb{R}^{n}\right)$ if and only if $f \in L^{p}\left(\mathbb{R}^{n}\right)$ and $\mathcal{D}_{\alpha, m, c, q}(f) \in L^{p}\left(\mathbb{R}^{n}\right)$, where $c=-\frac{2 \pi^{2}}{(2 \pi)^{\alpha}(n+2)}$.

Theorem 4.8. Let $p, q \in(1, \infty)$ and $\alpha \in[2,4)$. Then $f \in B_{p, q}^{m \alpha}\left(\mathbb{R}^{n}\right)$ if and only if $f \in L^{p}\left(\mathbb{R}^{n}\right)$ and $\mathcal{D}_{\alpha, m, c, q}(f) \in L^{p}\left(\mathbb{R}^{n}\right)$, where $c=-\frac{2 \pi^{2}}{(2 \pi)^{\alpha}(n+2)}$. 


\section{Characterizations $F_{p, q}^{\alpha}$ and $B_{p, q}^{\alpha}$ using $S_{E, \alpha, q}$}

Throughout this section, $E$ denotes a nonzero, bounded radial function on $\mathbb{R}^{n}$ with compact support and it satisfies

$$
\int_{\mathbb{R}^{n}} E(x) d x=1
$$

Let $1<q<\infty$ and $\alpha>0$, the Littlewood-Paley type function $S_{E, \alpha, q}$ is defined by

$$
S_{E, \alpha, q}(f)(x)=\left(\int_{0}^{\infty}\left|\frac{E_{t} * f(x)-f(x)}{t^{\alpha}}\right|^{q} \frac{d t}{t}\right)^{1 / q} .
$$

Noting that $\chi_{B}$ in Theorem 1.1 is a special function $E$ satisfying the above assumptions, Sato et al. in [18] (see also [29]) characterized Sobolev spaces $W^{\alpha, p}\left(\mathbb{R}^{n}\right)$ with $\alpha \in(0,2)$ and $p \in(1, \infty)$ by using the square function

$$
S_{E, \alpha, 2}(f)(x)=\left(\int_{0}^{\infty}\left|\frac{E_{t} * f(x)-f(x)}{t^{\alpha}}\right|^{2} \frac{d t}{t}\right)^{1 / 2} .
$$

Theorem 5.1 ([18]). Let $p \in(1, \infty)$ and $\alpha \in(0,2)$. If $E$ is nonnegative, then $f \in W^{\alpha, p}\left(\mathbb{R}^{n}\right)$ if and only if $f \in L^{p}\left(\mathbb{R}^{n}\right)$ and $S_{E, \alpha}(f) \in L^{p}\left(\mathbb{R}^{n}\right)$.

Chen et al. in [5, Theorem 4.1, p. 291] further improved Theorem 5.1 by removing the nonnegative assumption on the function $E$.

Theorem 5.2 ([5]). Let E be a nonzero, bounded radial function on $\mathbb{R}^{n}$ with compact support and satisfy

$$
\int_{\mathbb{R}^{n}} E(y) d y=1
$$

For $p \in(1, \infty)$ and $a \in(0,2)$, we have $f \in W^{\alpha, p}\left(\mathbb{R}^{n}\right)$ if and only if $f \in L^{p}\left(\mathbb{R}^{n}\right)$ and $S_{E, \alpha, 2}(f) \in$ $L^{p}\left(\mathbb{R}^{n}\right)$.

Additionally, if there exists an positive integer $N$ such that

$$
\int_{\mathbb{R}^{n}} E(y) y_{j}^{2 \ell} d y=0
$$

for all $j=1, \cdots, n$ and $\ell=1, \cdots, N$. Then we have $f \in W^{\alpha, p}\left(\mathbb{R}^{n}\right)$ if and only if $f \in L^{p}\left(\mathbb{R}^{n}\right)$ and $S_{E, \alpha, 2}(f) \in L^{p}\left(\mathbb{R}^{n}\right)$ for $a \in(0,2 N+2)$ and $p \in(1, \infty)$.

By checking the proof of Theorem 5.2, and using Theorems 2.1 and 2.2, we conclude that

Theorem 5.3. Let $1<q<\infty$ and let $E$ be as in Theorem 5.2. For $p \in(1, \infty)$ and $a \in(0,2)$, we have

(i) $f \in F_{p, q}^{\alpha}\left(\mathbb{R}^{n}\right)$ if and only if $f \in L^{p}\left(\mathbb{R}^{n}\right)$ and $S_{E, \alpha, q}(f) \in L^{p}\left(\mathbb{R}^{n}\right)$; 
(ii) $f \in B_{p, q}^{\alpha}\left(\mathbb{R}^{n}\right)$ if and only if $f \in L^{p}\left(\mathbb{R}^{n}\right)$ and $S_{E, \alpha, q}(f) \in L^{p}\left(\mathbb{R}^{n}\right)$.

Additionally, if there exists an positive integer $N$ such that

$$
\int_{\mathbb{R}^{n}} E(y) y_{j}^{2 \ell} d y=0
$$

for all $j=1, \cdots, n$ and $\ell=1, \cdots, N$. Then we have

(i) $f \in F_{p, q}^{\alpha}\left(\mathbb{R}^{n}\right)$ if and only if $f \in L^{p}\left(\mathbb{R}^{n}\right)$ and $S_{E, \alpha, q}(f) \in L^{p}\left(\mathbb{R}^{n}\right)$ for $a \in(0,2 N+2)$ and $p \in(1, \infty)$;

(ii) $f \in B_{p, q}^{\alpha}\left(\mathbb{R}^{n}\right)$ if and only if $f \in L^{p}\left(\mathbb{R}^{n}\right)$ and $S_{E, \alpha, q}(f) \in L^{p}\left(\mathbb{R}^{n}\right)$ for $a \in(0,2 N+2)$ and $p \in(1, \infty)$.

\section{Acknowledgements}

The research was supported by National Natural Science Foundation of China (Grant Nos. 11971295, 11871108 and 11871436) and Natural Science Foundation of Shanghai (No. 19ZR1417600).

\section{References}

[1] R. Alabern, J. Mateu, and J. Verdera, A new characterization of Sobolev spaces on $\mathbb{R}^{n}$, Math. Ann., 354(2) (2012), 589-626.

[2] J. Bourgain, Averages in the plane over convex curves and maximal operators, J. Anal. Math., 47 (1986), 69-85.

[3] D. Chang, J. Liu, D. Yang, and W. Yuan, Littlewood-Paley characterizations of HajłaszSobolev and Triebel-Lizorkin spaces via averages on balls, Potential Anal., 46(2) (2017), 227259.

[4] D. Chang, D. Yang, W. Yuan, and J. Zhang, Some recent developments of high order Sobolevtype spaces, J. Nonlinear Convex Anal., 17(9) (2016), 1831-1865.

[5] J. Chen, D. Fan, and F. Zhao, Littlewood-Paley functions and Sobolev spaces, Nonlinear Anal., 184 (2019), 273-297.

[6] F. Dai, A. Gogatishvili, D. Yang, and W. Yuan, Characterizations of Sobolev spaces via averages on balls, Nonlinear Anal., 128 (2015), 86-99.

[7] F. Dai, A. Gogatishvili, D. Yang, and W. Yuan, Characterizations of Besov and TriebelLizorkin spaces via averages on balls, J. Math. Anal. Appl., 433(2) (2016), 1350-1368.

[8] F. Dai, J. Liu, D. Yang, and W. Yuan, Littlewood-Paley characterizations of fractional Sobolev spaces via averages on balls, Proc. Roy. Soc. Edinburgh Sect. A, 148(6) (2018), 1135-1163.

[9] Y. Ding, D. Fan, and Y. Pan, On Littlewood-Paley functions and singular integrals, Hokkaido Math. J., 29(3) (2000), 537-552.

[10] R. Fefferman, A note on singular integrals, Proc. Amer. Math. Soc., 74(2) (1979), 266-270.

[11] M. Frazier and B. Jawerth, Decomposition of Besov spaces, Indiana Univ. Math. J., 34(4) (1985), 777-799.

[12] M. Frazier, and B. Jawerth, A discrete transform and decompositions of distribution spaces, J. Funct. Anal., 93(1) (1990), 34-170. 
[13] A. Gogatishvili, P. Koskela and Y. Zhou, Characterizations of Besov and Triebel-Lizorkin spaces on metric measure spaces, Forum Math., 25(4) (2013), 787-819.

[14] L. Grafakos, Modern Fourier Analysis, Second edition, Graduate Texts in Mathematics 250, Springer, New York, 2009.

[15] T. Heikkinen, L. Ihnatsyeva and H. Tuominen, Measure density and extension of Besov and Triebel-Lizorkin functions, J. Fourier Anal. Appl., 22(2) (2016), 334-382.

[16] T. Runst, and W. Sickel, Sobolev spaces of Fractional Order, Nemytskij Operators, and Nonlinear Partial Differential Equations, De Gruyter Series in Nonlinear Analysis and Applications 3, Walter de Gruyter Co., Berlin, 1996.

[17] W. Sickel, and H. Triebel, Hölder inequalities and sharp embeddings in function spaces of $B_{p q}^{s}$ and $F_{p q}^{s}$ type, Z. Anal. Anwendungen, 14(1) (1995), 105-140.

[18] S. Sato, F. Wang, D. Yang, and W. Yuan, Generalized Littlewood-Paley characterizations of fractional Sobolev spaces, Commun. Contemp. Math., 20(7) (2018), 1750077.

[19] E. M. Stein, On the functions of Littlewood-Paley, Lusin, and Marcinkiewicz, Trans. Amer. Math. Soc., 88 (1958), 430-466.

[20] E. M. Stein, Singular Integral and Differential Properties of Functions, Princeton University Press, Princeton, N.J., 1970.

[21] E. M. Stein, Maximal functions: Spherical means, Proc. Nat. Acad. Sci. U.S.A., 73(7) (1976), 2174-2175.

[22] E. M. Stein, Harmonic Analysis: Real-Variable Methods, Orthogonality, and Oscillatory Integrals, Princeton Mathematical Series 43, Monographs in Harmonic Analysis, III, Princeton University Press, Princeton, N.J., 1993.

[23] H. Triebel, Theory of Function Spaces, Monographs in Mathematics 78, Birkhäuser Verlag, Basel, 1983.

[24] H. Triebel, Theory of Function Spaces, II, Monographs in Mathematics 84, Birkhäuser Verlag, Basel, 1992.

[25] D. Yang, and W. Yuan, Pointwise characterizations of Besov and Triebel-Lizorkin spaces in terms of averages on balls, Trans. Amer. Math. Soc., 369(11) (2017), 7631-7655.

[26] D. Yang, W. Yuan, and Y. Zhou, A new characterization of Triebel-Lizorkin spaces on $\mathbb{R}^{n}$, Publ. Mat., 57(1) (2013), 57-82.

[27] W. Yuan, W. Sickel, and D. Yang, Morrey and Campanato Meet Besov, Lecture Notes in Mathematics 2005, Springer-Verlag, Berlin, 2010.

[28] J. Zhang, C. Zhuo, D. Yang, and Z. He, Littlewood-Paley characterizations of TriebelLizorkin-Morrey spaces via ball averages, Nonlinear Anal., 150 (2017), 76-103.

[29] Y. Zhang, D. Chang, and D. Yang, Generalized Littlewood-Paley characterizations of TriebelLizorkin spaces, J. Nonlinear and Convex Anal., 18(6) (2017), 1171-1190.

[30] C. Zhuo, W. Sickel, D. Yang, and W. Yuan, Characterizations of Besov-type and TriebelLizorkin-type spaces via averages on balls, Canad. Math. Bull., 60(3) (2017), 655-672. 